\title{
Autistic Traits Associated With Food Neophobia But Not
}

\section{Olfactory Sensitivity}

Correspondence to be sent to: Lorenzo D. Stafford, Department of Psychology, University of Portsmouth, King Henry Building, King Henry I Street, Portsmouth PO1 2DY.U.K. Email: lorenzo.Stafford@port.ac.uk. Tel: 02392846322. Fax: 02392846300 


\section{Abstract}

2

3 Food neophobia has been shown to be associated with a range of personality traits (including

4 anxiety, lower sensation seeking) and additionally sensory aspects of food such as taste and

5 texture. Running parallel to that work, research has demonstrated higher incidences of food

6 neophobia in autistic populations and separately evidence of hypersensitivity in some sensory

7 domains. The aim of the current study was to extend our understanding by exploring whether

8 the broader aspects of autistic traits can predict food neophobia in a non-autistic population and

9 whether this is mediated by differences in olfactory sensitivity. In the present study, student

10 participants $(\mathrm{N}=50)$ completed questionnaires measuring their food neophobia (FNS) and

11 preferences for foreign cuisine, autistic traits (Autistic Quotient, AQ), and then completed an

12 olfactory threshold test for a food related odour. The findings demonstrated a positive

13 association between food neophobia and the magnitude of autistic traits and interestingly, an

14 inverse relation between preference for foreign cuisine and olfactory sensitivity; those

15 individuals less inclined toward foreign cuisine had poorer sensitivity to a food related odour.

16 Since AQ was not related to olfactory sensitivity, these findings suggest the relation between

17 autistic traits and food neophobia is unlikely to be mediated by olfactory sensitivity. More

18 broadly however, our sense of smell is associated with experiencing a wider diet.

\section{Keywords}

20 Autism, Olfaction, Food Neophobia, Odor 


\section{Introduction}

2 The reluctance to try new foods is commonly referred to as food neophobia (Birch et al., 1987)

3 and is thought to reflect the tension in all humans of, on the one the hand, avoiding novel foods

4 that may harm us and on the other, approaching novel foods that confer advantages of a wider

5 diet (Pliner and Hobden, 1992). Food neophobia can be measured using instruments such as the

6 Food Neophobia Scale (FNS, Pliner and Hobden, 1992), where individuals respond to questions

7 on their propensity to try novel foods in different situations. Research has shown that younger

8 children tend to be more food neophobic (Birch, 1999) and generally appears to reduce with age

9 (Otis, 1984). Work has also found that in young children, those with psychological and

10 behavioural problems are less likely to try novel foods (Pelchat and Pliner, 1986). Of particular

11 interest to the present study is the influence of behavioural ridgity in restricted eating habits,

12 which can be manifested by rituals in preparation and presentation (Jacobi et al., 2008). These

13 factors are also indicative to those on the Autistic spectrum, and research has shown that

14 childhood food neophobia is higher in those populations (Bandini et al., 2010; Barnhill et al.,

15 2015). However, what remains unclear is to what extent these sorts of traits (i.e. behavioural

16 ridgity) relate to food neophobia in non-clinical adult populations. This is important since

17 personality traits such as low sensation seeking (that also relate to autistic traits, Romero-

18 Martínez, et al., 2015) can predict lower preference for trying new foods (Frank \& van der

19 Klaauw, 1994), hence it could be that those sorts of traits are part of a wider personality

20 constellation of autistic related tendencies.

22 Food neophobia is also driven by sensory factors such as the smell, taste and texture of the food

23 (Wildes et al., 2012). For instance, research in adults has shown that those individuals less 
1 willing to try new foods rated a selection of odours as less pleasant and less intense and in a

2 measure of 'ideal' taste intensities, preferred lower levels of intensity (Frank \& van der Klaauw,

3 1994). This suggests that there are differences in odour hedonics and sensitivity in food

4 neophobics. Separately and apart from food research, olfactory abilities in autistic versus non-

5 autistic populations have shown either impairments (Suzuki et al., 2003), or no differences

6 (Tavassoli and Baron-Cohen, 2012). Those findings are surprising as one might reason that

7 given the evidence for hypersensitivity in other modalities (e.g. Vision: Simmons et al., 2009;

8 Audition: Jones et al., 2009) that oversensitivity to odours would be a characteristic of autistic

9 populations and by extension offer some evidence as to their role in the wider possible

10 relationship between autistic traits and food neophobia. However, in both of the autistic/non-

11 autistic lines of research, there is a mismatch between the types of olfactory tests employed and

12 whether food related odours were utilized. For this reason, the present study used a reliable and

13 widely used test of olfactory sensitivity (Threshold detection test) together with a test of

14 hedonics (pleasantness) and intensity; and importantly, we used a food related odour.

16 In the current study, individuals completed questionnaires measuring their food neophobia (FNS)

17 and preferences for foreign cuisine, with both measures found to correlate strongly in the original

18 study (Pliner and Hobden, 1992). We included the latter as we are interested in differences in

19 the sense of smell and how this relates to behavioural aspects (Autistic traits) and attitudes

20 toward food. In contrast to the FNS, attitudes toward different types of food cuisine offers a

21 more tangible measure of food neophobia, particularly relevant to these other characteristics.

22 Individuals then completed a measure on the degree to which they present autistic traits (Autistic

23 Quotient, AQ); followed by a test of olfactory sensitivity for a food related odour. It was 
1 expected that Food neophobia and rejection (i.e. less preference) for foreign cuisine would be

2 positively associated with AQ and we tentatively predicted that both of these would relate to

3 increases in olfactory sensitivity.

4

5

6

7

8 Method

9

10 Participants

11 Fifty University students, aged between 18 and 24 (40 females) participated in the study ( $M=$

1220.4 years, $\mathrm{SD}=4.2$ years). Participants were recruited using an online system where the study

13 was advertised as examining factors that influence our sense of smell. Participants with

14 respiratory conditions (i.e. asthma) were advised not to take part due to possible adverse effects.

15 All participants reported good health and free from any colds that may have affected their ability

16 to smell. Participants gave written informed consent and the study protocol was given ethical

17 approval from the department's ethics committee (British Psychology Society guidelines,

18 consistent with the declaration of Helsinki).

19

20 Design

21 The study used a regression design exploring the following variables: Autistic Quotient (AQ),

22 Food Neophobia Scale (FNS), Preference for Foreign Cuisine (PFC) and olfactory measures

23 (threshold/intensity/pleasantness).

24

25 
12 neophobia.

13

24 scale can range from 3-21.

\section{Materials}

Food Neophobia Scale (FNS)

The FNS consisted of ten items (Pliner and Hobden, 1992). This measured the likelihood of avoiding new foods in different situations. Responses were made on a Likert scale of 1 (Strongly disagree) to 7 (Strongly agree) for all items. Half of the items were positive statements towards food such as "I am constantly sampling new and different foods." The other half consisted of negative statements towards food such as "I am afraid to eat things I have never had before."

Ratings from the positive items (1, 4, 6, 9 and 10) were reversed and the sum was calculated to form FNS score. Scores can range from 10 -70, with higher scores indicating higher food

\section{Preference For Foreign Cuisine (PFC)}

Following the FNS were three additional questions that asked how often they ate foods from different countries (Chinese, Italian and Indian food). The questions were adapted from Pliner and Hobden (1992) and were found by those authors to correlate strongly with the FNS. The three cuisines were chosen as they are the most common foreign cuisine consumed in the UK. The question was “How often do you eat 'nnn' food?”, where 'nnn' was replaced with the relevant cuisine (Chinese/Italian/Indian, fixed order). Responses were made on a scale of 1 (never) to 7 (frequently). All ratings were reversed individually before summing the score, so that a high score showed that they consumed food from those cuisines less often; This scoring facilitated the comparison with other factors and in particular with the FNS. The scores on this 
2 The AQ used was the original version for aged 16+ devised by Baron-Cohen et al., (2001) which

3 is used to measure the level of autistic tendencies. It consisted of 50 questions made up of 10

4 questions covering 5 different areas: social skill (e.g. "I find social skills easy" - reversal

5 scored), attention switching (for example "I frequently get so strongly absorbed in one thing that

6 I lose sight of other things"), attention to detail (e.g. "I tend to notice things that others do not"),

7 communication (e.g. "I enjoy social chit chat" - reversal scored) and imagination (for example "I

8 find making up stories easy" - reversal item). The responses for all questions were: definitely

9 agree, slightly agree, slightly disagree and definitely disagree, 1 point was scored when the

10 response was either definitely/slightly in the autistic behaviour items. Any other responses were

11 scored 0 . The sum of this score was the final AQ score. The scores can range from $0-50$. A high

12 score represents a high number of autistic tendencies.

13

14

15

16

17

\section{Olfactory Threshold Test}

The odour used for the threshold test was a sweet smelling chocolate odourant (Code 0679, Anglo brands, UK) used in previous work (Stafford and Whittle, 2015) which was diluted in propylene glycol (Fisher Scientific, UK). The odourant was prepared using sixteen 250ml squeeze bottles, in 16 dilution steps, starting at $0.125 \%$ (Step 1) with each successive step diluted by a factor of two using serial dilution to the lowest (Step 16) dilution. In addition to the odour containing bottles, for each dilution step, two 'blank' squeeze bottles (containing dilutant only) were used in the threshold test. Testing commenced by asking participants to smell the bottle with the highest concentration to familiarise themselves with the target odour. They were then presented with the triplet containing the weakest concentration. Following presentation of the 
1 last bottle of the triplet (counterbalanced), participants were asked which bottle contained the

2 odour (1,2 or 3). If the participant answered correctly (and it was the lowest concentration),

3 they were presented with the same triplet again (in a different order) and the task repeated until

4 they made a mistake, which resulted in the triplet containing the next concentration step being

5 presented. Using a single up-down staircase system, this was then repeated until there were

6 seven 'turning points', with the mean of the last four points determining the threshold for the

7 individual. Each bottle was held under the participant's nose $(\approx 2 \mathrm{~cm})$ and gently waved between

8 each nostril to ensure optimal inhalation. A blindfold was used by the participants to avoid

9 odour identification. The experimenter wore cotton gloves (Boots the Chemist,) to reduce any

10 cross contamination of odours.

12 Procedure

13 Prior to the experiment, participants were instructed not to consume anything apart from water

14 two hours before the experiment, this also included chewing gum, sweets and cigarettes. They

15 were also told not to wear any perfume/aftershave or use any other strong smelling soaps, to

16 ensure they were only smelling the odourant. The study took place within the University's

17 Department of Psychology. The room used for the study was large and well ventilated to avoid

18 other odours in the room affecting the results of the odour test. Participants completed an

19 informed consent form followed by ratings of how hungry they felt using a Visual Analogue

20 Scale (VAS) of a 100mm unmarked line labelled "not at all" and "extremely" at either end.

21 Hunger ratings were taken as some previous research has shown that level of hunger can

22 influence olfactory sensitivity (Stafford and Welbeck, 2010). They then completed the FNS and

23 PFC, followed by the AQ. Next, they rated the intensity and pleasantness of the chocolate odour

24 using Visual Analogue Scales (VAS), followed by the threshold test. On completion of this test, 
1 participants were given a full debriefing and thanked for their time.

\section{Data Analyses}

7 Preliminary analyses revealed that hunger ratings were not significantly related to olfactory

8 threshold score and were therefore excluded from further analyses. We then completed

9 hierarchical linear regression analyses separately for FNS and PFC as criterion variables. For

10 each of these analyses, the same predictor variables were entered using blockwise entry, where in

11 step 1, we used AQ, step 2: odour threshold, step 3: odour intensity, step 4: odour pleasantness.

12 The rationale for this order was based on the study's main areas of interest together with

13 previous research (e.g. Ashwin et al., 2014). The data were also checked to ensure they met the

14 assumptions for regression analyses. Finally, since it was important to examine the relationship

15 between AQ and olfactory threshold separately to their predictive utility, we completed Pearson

16 correlation analysis. The same method of analysis was used to test the relationship between FNS

17 and PFC as a way of validating that these two variables were measuring similar aspects of food

18 attitudes. 
11 For FNS, analyses revealed a significant positive association for AQ (Table 2), where as

12 predicted, increases of AQ were associated with greater food neophobia. The association

13 between FNS and Odour pleasantness approached significance $(\mathrm{p}=.09)$ and increased the

14 predictive utility of the final model by the same magnitude. Against prediction, there was no 15 significant association between FNS and odour threshold.

17 In contrast, the regression for PFC demonstrated that olfactory threshold was negatively

18 associated with this measure $(\mathrm{p}=.002)$ (Table 3$)$, suggesting that increases in olfactory

19 sensitivity were associated with increasing preference (i.e. lower dislike) for foreign cuisine.

21 We completed correlational analyses to understand the relationship between AQ and Olfactory

22 threshold and separately FNS and PFC. For the former, against our prediction, this revealed no

23 significant relation, $\mathrm{r}(50)=.11, \mathrm{p}=.43$, whereas the expected association between FNS and PFC 24 was significant, $\mathrm{r}(50)=.33, \mathrm{p}=.02$. 


\section{Discussion}

2 We found that increases in AQ scores were associated with increases in food neophobia, which

3 supports the prediction that people with higher autistic traits are more reluctant to try new foods.

4 This finding extends previous work in children and young adults with autism (Beighley et al.,

5 2013; Kuschner et al., 2015) by demonstrating that in the wider adult population, autistic traits

6 are associated with higher food neophobia. To date, increased food neophobia has been

7 associated with a range of personality measures including higher trait anxiety; lower sensation

8 seeking (Pliner \& Hobden, 1992; Frank \& van der Kaauw, 1994). Separately, research has

9 demonstrated that both trait anxiety and low sensation seeking are also related to increases in AQ

10 (Horder et al., 2014; Romero-Martínez et al., 2015). Since we found in the present study that

11 both food neophobia and autistic tendencies are positively related in a non-autistic population,

12 this suggests that this is part of a wider behavioural trait, where those more inclined toward

13 aspects including behavioural rigidity, have a narrower dietary repertoire. In terms of the wider

14 implications of this finding, it may be of applied importance when looking at ways of changing

15 attitudes toward food neophobia, including children; whereby in addition to existing

16 interventions (Wardle et al., 2003) it might be more fruitful to adapt some aspects from the

17 autistic research domain, including individualized reinforcement interventions (Koegel et al., 18 2012).

19 We also found that preference for foreign cuisine was inversely related to olfactory sensitivity;

20 individuals exhibiting lower preference for foreign cuisine had poorer olfactory sensitivity. To

21 our knowledge, this is a novel finding and concords with work where odour identification was

22 poorer in those individuals categorised as food neophobic (Dematte et al., 2013). This could be

23 be related to a reduced sniff magnitude in those individuals less willing to try novel foods, as has 
1 been found in previous work (Raudenbush et al., 1998). In these populations, this could be part

2 of a reduced desire (or indeed heightened protection) in smelling unfamiliar foods (Raudenbush

3 et al., 1995). In the present study, the consequence of less engagement with sniffing the test

4 odour would likely be poorer performance in the odour detection test, as suggested in some

5 previous work (see review Mainland \& Sobel, 2006). From the opposite perspective, as

6 theorised elsewhere (Dematte et al., 2013), it could be that individuals who were more likely to

7 try novel food cuisines would necessarily sample more food related odours and on that basis be

8 more accurate in food odour identification. Of course, as with the current study, one cannot rule

9 out the possibility that rather than food neophobia directly influencing odour performance it

10 could be that those that have a better sense of smell are less food neophobic; hence the direction

11 of the association is unclear. Evidence is therefore needed that some period of exposure or

12 training leads to a demonstrably better sense of smell. In this vein, one study has shown that

13 students of wine tasting had better identification for a range of general odourants compared to

14 controls (Marino-Sanchez et al., 2010). In earlier work, we also found that individuals'

15 performance on an odour threshold test similar to the current study, improved on a second

16 session (Stafford and Welbeck, 2010). These two studies suggest that greater exposure or odour

17 training may increase odour performance. This also chimes with animal work where mice

18 exposed to an odour during an active learning phase showed greater activity in the olfactory

19 related regions of the brain (Abraham et al., 2014).

20 It also needs to be acknowledged that the finding in the present study was specifically between

21 neophobia for 'foreign cuisine' and olfactory sensitivity, whereas the previous study's finding

22 was between the FNS and odour identification (Dematte et al., 2013). Since we also found that

23 the two scales (FNS/PFC) were correlated in the current study, it is unclear why a similar 
1 FNS/olfactory sensitivity association was absent. One possibility is that, had we used a larger

2 sample as that work $(\mathrm{n}=167)$ with more variation in FNS scores, the association would have

3 reached statistical significance. Additionally, it is important to recognise that the finding itself

4 be treated with caution, since the measure is based on foreign cuisines (Chinese/Italian/Indian)

5 that are in fact common here in the UK and therefore limiting their usefulness as a measure of

6 'novel' types of food. Interestingly however, the range of responses on this measure did show

7 reasonable variability [from high preference (3) to low preference (18)] which is essential for any

8 measure; and additionally, the scale did correlate with FNS. It also needs to be considered that

9 selecting more unusual cuisines (e.g. Moroccan, Vietnamese) might run the risk of floor effects

10 with minimal variability. Nevertheless, achieving a balance between low/high novel cuisines

11 would be useful in future research.

13 The finding that AQ scores did not relate to olfactory sensitivity was interesting and consistent

14 with some previous work (Tavassoli and Baron-Cohen, 2012) that compared sensitivity to a non-

15 food odour between individuals with ASC and controls. Interestingly however, one study did find in

16 a ASC sample $(\mathrm{n}=17)$ that AQ scores were positively associated with greater olfactory performance

17 (Ashwin et al., 2014). In that work, performance was measured using the Alcohol Sniff Test, where

18 the distance at which the odour could be detected was the dependent variable. It is therefore possible

19 that the contrasting findings are explained by the different olfactory tests used. Nevertheless, given

20 that the olfactory test used in the current study is a more standardised and widely used measure of

21 olfactory sensitivity (Hummel et al., 2007), together with the use of a food related odour, all suggest

22 that olfactory sensitivity is unlikely to play a central role in the association between AQ and food

23 neophobia; though clearly more work is needed to support this theory. 
1 In terms of study limitations, since the study here used a predominantly female sample, it is

2 unclear if the same results would be seen with a larger number of males. Given that ASC is

3 more prevalent in males (Fombonne et al., 2011), it is possible that males' AQ scores would be

4 higher than females and provide a greater range and ability to examine the questions in the

5 present study. Interestingly however, in the study here, the comparison of AQ scores between

6 males and females was not significant $(\mathrm{p}>.35)$, making that possibility unlikely. Related to this

7 point, it needs to be acknowledged in the present study, that although there was good variability

8 in the data, none of the individuals' AQ scores reached the level for diagnoses and hence future

9 research is recommended to see if the present findings apply equally to a wider sample.

11 In summary, we found that in a student sample that increases in autistic traits (AQ) were

12 associated with higher food neophobia (FNS) and that olfactory sensitivity was greater for those

13 with a greater preference (less neophobic) for foreign cuisines. These findings point toward the 14 importance of further research on autism and dietary preferences. 

plasticity of sensory inputs mediated by olfactory learning. Elife, 3, e02109.

Ashwin C, Chapman E, Howells J, Rhydderch D, Walker I, and Baron-Cohen S (2014) Enhanced olfactory sensitivity in autism spectrum conditions. Molecular autism, 5(1), 53.

Baron-Cohen S, Wheelwright S, Skinner R, Martin J and Clubley E (2001) The autism-spectrum quotient (AQ): Evidence from asperger syndrome/high-functioning autism, males and females, scientists and mathematicians. Journal of autism and developmental disorders, 31(1), 5-17.

Bandini LG, Anderson SE, Curtin C, Cermak S, Evans EW, Scampini R, Maslin M, Must A (2010) Food selectivity in children with autism spectrum disorders and typically developing children. The Journal of pediatrics, 157(2), 259-264.

Barnhill K, Gutierrez A, Marti SN and Hewitson L (2015) Analysis of Dietary Intake and Nutritional Status in Children with Autism Spectrum Disorder. Autism Open Access, 5(1000154), 2. doi: $10.4172 / 2165-7890.1000154$

Beighley JS, Matson JL, Rieske RD and Adams HL (2013) Food selectivity in children with and without an autism spectrum disorder: Investigation of diagnosis and age. Research in developmental disabilities, 34(10), 3497-3503. doi:10.1016/j.ridd.2013.07.026

Birch LL, McPhee L, Shoba BC, Pirok E and Steinberg L (1987) What kind of exposure reduces children's food neophobia?: Looking vs. tasting. Appetite, 9(3), 171-178.

Birch LL (1999) Development of food preferences. Annual review of nutrition, 19(1), 41-62.

Demattè ML, Endrizzi I, Biasioli F, Corollaro ML, Pojer N, Zampini M, Aprea E, Gasperi F (2013) Food neophobia and its relation with olfactory ability in common odour identification. Appetite, 68, 112-117. doi:10.1016/j.appet.2013.04.021

Fombonne E, Quirke S and Hagen A (2011) Epidemiology of pervasive developmental disorders. In Amaral D.G., Dawson G. and Geschwind D.H. eds. (2011) Autism spectrum disorders. New York: Oxford University Press, pp. $90-111$.

Frank, R. A., \& van der Klaauw, N. J. (1994). The contribution of chemosensory factors to individual differences in reported food preferences. Appetite, 22(2), 101-123.

Hummel T, Kobal G, Gudziol H, Mackay-Sim A (2007) Normative data for the "Sniffin' Sticks" including tests of odor identification, odor discrimination, and olfactory thresholds: an upgrade based on a group of more than 3,000 subjects. European Archives of Oto-Rhino-Laryngology, 264, 237-243. 
Horder, J., Wilson, C. E., Mendez, M. A., \& Murphy, D. G. (2014). Autistic traits and abnormal sensory experiences in adults. Journal of Autism and Developmental Disorders, 44(6), 14611469.

Jacobi, C., Schmitz, G., \& Agras, W. S. (2008). Is picky eating an eating disorder?. International Journal of Eating Disorders, 41(7), 626-634.

Jaeger, S. R., Rasmussen, M. A., \& Prescott, J. (2017). Relationships between food neophobia and food intake and preferences: Findings from a sample of New Zealand adults. Appetite, 116, 410-422

Jones CR, Happé F, Baird G, Simonoff E, Marsden AJ, Tregay J, and Charman T (2009) Auditory discrimination and auditory sensory behaviours in autism spectrum disorders. Neuropsychologia, 47(13), 2850-2858. doi:10.1016/j.neuropsychologia.2009.06.015

Koegel, R. L., Bharoocha, A. A., Ribnick, C. B., Ribnick, R. C., Bucio, M. O., Fredeen, R. M., \& Koegel, L. K. (2012). Using individualized reinforcers and hierarchical exposure to increase food flexibility in children with autism spectrum disorders. Journal of autism and developmental disorders, 42(8), 1574-1581.

Kuschner ES, Eisenberg IW, Orionzi B, Simmons WK, Kenworthy L, Martin A and Wallace GL (2015) A preliminary study of self-reported food selectivity in adolescents and young adults with autism spectrum disorder. Research in Autism Spectrum Disorders, 15, 53-59. doi:10.1016/j.rasd.2015.04.005

Mainland, J., \& Sobel, N. (2006). The sniff is part of the olfactory percept. Chemical senses, 31(2), 181-196.

Mariño-Sánchez FS, Alobid I, Centellas S, Alberca C, Guilemany JM, Canals JM, De Haro J, Mullol J (2010) Smell training increases cognitive smell skills of wine tasters compared to the general healthy population. The WINECAT Study. Rhinology, 48(3), 273.

Otis L (1984) Factors influencing the willingness to taste unusual foods. Psychological Reports, 54, 739-745.

Pelchat M and Pliner P (1986) Antecedents and correlates of feeding problems in young children. Journal of Nutrition Education, 18, 23-29.

Pliner P and Hobden K (1992) Development of a scale to measure the trait of food neophobia in humans. Appetite, 19(2), 105-120.

41 
Raudenbush, B., Schroth, F., Reilley, S., \& Frank, R. A. (1998). Food neophobia, odor evaluation and exploratory sniffing behavior. Appetite, 31(2), 171-183.

Romero-Martínez, Á., Moya-Albiol, L., Vinkhuyzen, A. A., \& Polderman, T. J. (2015). Genetic and environmental contributions to the inverse association between specific autistic traits and experience seeking in adults. American Journal of Medical Genetics Part B: Neuropsychiatric Genetics.

Simmons DR, Robertson AE, McKay LS, Toal E, McAleer P, and Pollick FE (2009) Vision in autism spectrum disorders. Vision research, 49(22), 2705-2739.

Stafford LD, and Welbeck K (2011) High hunger state increases olfactory sensitivity to neutral but not food odors. Chemical senses, 36(2), 189-198.

Stafford LD, and Whittle A (2015) Obese individuals have higher preference and sensitivity to odor of chocolate. Chemical senses, O(0), 1-6 doi:10.1093/chemse/bjv007

Suzuki Y, Critchley HD, Rowe A, Howlin P and Murphy DG (2003) Impaired olfactory identification in Asperger's syndrome. The Journal of neuropsychiatry and clinical neurosciences, 15(1), 105.

Tavassoli T, and Baron-Cohen S (2012) Olfactory detection thresholds and adaptation in adults with autism spectrum condition. Journal of autism and developmental disorders, 42(6), 905909.doi: 10.1007/s10803-011-1321-y

Wardle, J., Herrera, M. L., Cooke, L., \& Gibson, E. L. (2003). Modifying children's food preferences: the effects of exposure and reward on acceptance of an unfamiliar vegetable. European journal of clinical nutrition, 57(2), 341-348.

Wildes, J. E., Zucker, N. L., \& Marcus, M. D. (2012). Picky eating in adults: Results of a webbased survey. International Journal of Eating Disorders, 45(4), 575-582. 
1 Table 1. Mean Participant Characteristics

\begin{tabular}{cccc}
\hline & Mean & SD & Range \\
\hline Age & 20.4 & 4.2 & 18 \\
FNS & 31.1 & 12.1 & 50 \\
PFC & 9.6 & 3.7 & 15 \\
AQ & 17.1 & 5.3 & 25 \\
Hunger & 38.8 & 27.9 & 96 \\
Threshold & 5.9 & 3.6 & 13.5 \\
& & & \\
Pleasantness & 65.5 & 21.9 & 85 \\
Intensity & 44.7 & 22.9 & 86 \\
& & & \\
FNS=Food Neophobia Scale; PFC=Preference for Foreign Cuisine; AQ=Autistic Quotient
\end{tabular}


Table 2. Effects Of AQ, Odour (Threshold, Intensity, Pleasantness) On Food Neophobia

Scale Scores

Variable $\quad$ R Square $\quad$ F Change $\quad$ Standardized $t$-value $p$-value
(beta) coefficients

\begin{tabular}{llllll}
\hline Model 1 & .20 & 11.87 & & .001 \\
AQ & & & .45 & 3.45 & .001
\end{tabular}

Model 2

.01

AQ

.45

.01

.001

Odour Threshold

$.22 \quad 1.06$

Model 3

AQ

.43

Odour Threshold

Odour Intensity

.002

.14

3.20

.92

001

.92

Model 4

.27

2.99

3.40
.11

.92

AQ

.43

.013

Odour Threshold

Odour Intensity

.13

.22

.01

.31

.002

.99

.31

Odour Pleasantness

1.03

.09

.002

3.30

.09

.92

.33

.99

.09

6

7

8

9 
Table 3. Effects Of AQ, Odour (Threshold, Intensity, Pleasantness) On Preference For

Foreign Cuisine

3

4

\begin{tabular}{cccc}
\hline Variable & F Square & $\begin{array}{c}\text { Standardized } \\
\text { (beta) } \\
\text { coefficients }\end{array}$ & $t$-value
\end{tabular}

Model 1
AQ

02

.74

.12

.40

AQ

.20

10.99

.86

.40

Model 2

AQ

Odour Threshold

.07

.56

.002

Model 3

AQ

Odour Threshold

Odour Intensity

.21

.33

$-3.31$

.58

$-.44$

.002

Model 4

AQ

Odour Threshold

Odour Intensity

Odour Pleasantness

\section{.21}

.06

.06
-.44
.08

.46

.57

$-.44$

$-3.30$

.65

.08

.57

.002

.57

$\begin{array}{ccc} & & .81 \\ .06 & .46 & .65 \\ -.44 & -3.28 & .002 \\ .08 & .56 & .58 \\ .03 & .24 & .81\end{array}$

5

6

7

8 